\title{
Alarm-Fatigue - wieviel Alarm verträgt der Mensch?
}

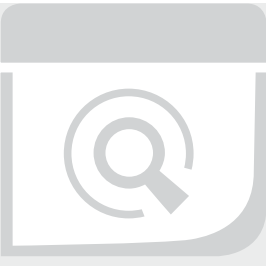

Durch zunehmende Technisierung in der Anästhesiologie und Intensivmedizin steigen sowohl Anzahl der Geräte als auch deren visuelle und akustische Alarme. Die meisten aller Alarme sind jedoch Fehlalarme, was bei den Mitarbeitern zu Frustration, Aggression und Fehlverhalten führt. Dieser Übersichtsartikel fasst die Risikofaktoren für die Entstehung von „Alarm-Fatigue“ zusammen und gibt einen Ausblick auf mögliche Lösungsstrategien.

\section{Einleitung}

Mitarbeiter von Intensivstationen hören bei ihrer täglichen Arbeit eine Vielzahl von Alarmen. Mit zunehmender Technisierung und Zunahme der Invasivität der Patientenüberwachung steigt die Anzahl von Alarmen und Fehlalarmen. Dies führt zu einer Desensibilisierung und damit zu einer Gefährdung der Patienten.

\section{GESCHICHTLICHES}

Der griechische Dichter Äsop (ca. 620-564 v. Chr.) berichtet in seiner Fabel „Der Junge, der Wolf schrie“ über einen Hirtenjungen, der vor den Toren der Stadt die Schafe hütet und aus Langeweile „Wolf“ ruft. Als ihm die Dorfbewohner zur Hilfe eilen, stellen sie fest, dass er sich nur einen Scherz erlaubte. Als einige Zeit später wirklich ein Wolf erscheint, bleibt der erneute Hilferuf des Hirten unbeantwortet, weil die Dorfbewohner ihm wieder einen Scherz unterstellen, und die Schafherde wird mitsamt des Hirten vom Wolf gefressen.

Die Moral dieser Fabel ist auch im deutschen Sprachraum bekannt: „Wer einmal lügt, dem glaubt man nicht, und wenn er auch die Wahrheit spricht".

\section{Bedeutung von Alarmen}

Im Operationssaal und auf der Intensivstation überwachen zahlreiche Medizingeräte die Lebensfunktionen der Patienten und erleichtern angemessene Behandlung wie auch Arbeit der Mitarbeiter. Nahezu jedes dieser Geräte vermag Alarme zu generieren. Alarme verkürzen idealerweise die Reaktionszeit zwischen dem Auftreten eines Problems und einer Intervention. Sie sind daher nicht nur unerlässlich für die Patientensicherheit, sondern können auch lebensrettend sein [1-3]. Alarme sind im klinischen Alltag weiterhin der Goldstandard [4].

Alarme sind in der Regel akustische Signale; zudem erscheint häufig gleichzeitig ein visueller Hinweis, der nach Definition des European Committee for Standardization (CEN, Comité Européen de Normalisation) mit zunehmender Priorität gelb aufleuchtet oder gelb bzw. rot blinkt [5]. Die aktuell gültige Version dieser Norm ist die BS EN 60601-1-8:2007+A1:2013. Das Ertönen eines Alarms dient dazu, die Aufmerksamkeit des Personals auf einen Patienten oder ein Gerät zu lenken, und zwar unabhängig davon, mit welcher Aufgabe das Personal momentan betraut ist. Sie erklingen, wenn z. B. voreingestellte Alarmgrenzen für eine gewisse Zeit über- oder unterschritten werden, das EKG einen pathologischen Rhythmus zu reflektieren scheint, ein Akku schwach oder ein Bett nicht an den Strom angeschlossen ist. Auch technische Alarme können vor potenziell lebensbedrohlichen Situationen warnen.

Verschiedene Studien zeigen, dass jeder Intensivpatient und dessen Behandlung mehr als 6 Alarme pro Stunde verursachen [6-8]. Die Mehrheit dieser Alarme, bis zu $90 \%$, ist jedoch falsch positiv, also ein Fehlalarm, führt dennoch oftmals zu einer Unterbrechung der Arbeit und begünstigt die Entstehung möglichen Fehlverhaltens von Mitarbeitern: Alarmgrenzen werden in kritische Bereiche verstellt, damit seltener ein Alarm ertönt, die Lautstärke wird so stark reduziert, dass der Alarm überhört werden kann, oder er wird gänzlich ignoriert [9]. Auf einer Intensivstation in einer Klinik in Salt Lake City [8] blieben $41 \%$ aller Alarme vom Personal völlig unbeantwortet, kein Mitarbeiter betrat nach dem Ertönen des Alarms das Patientenzimmer.

\section{Merke}

Die zahlreichen Alarme führen zu einer Desensibilisierung des Personals mit abnehmender oder verspäteter Beachtung [2, 3, 10-15], weil den Alarmen weniger Vertrauen geschenkt wird. Dies bezeichnet man als „Alarm-Fatigue“.

Die amerikanische Joint Commission on Accreditation of Healthcare Organizations, zuständig u.a. für die Zertifizierung amerikanischer Kliniken, warnt, dass eine Alarm-Fatigue die Patientensicherheit gefährdet [16]. Das Emergency Care Research Institute benennt 2 Jahre in Folge die Alarm-Fatigue als größte medizintechnologische Gefahr [17].

Eine verspätete Reaktion auf tatsächlich lebensgefährliche Situationen, die sich akustisch nicht von den anderen Fehlalarmen unterscheiden lassen, kann zu dramatischen Folgen führen. Die Joint Commission berichtete zwischen Januar 2009 und Juni 2012 von 98 alarmbedingten Zwischenfällen, die zu 80 Todesfällen führten und bei 13 Patienten bleibende Schäden hinterließen, mutmaßlich durch Alarm-Fatigue verursacht oder verstärkt [9]. Es ist aber davon auszugehen, dass die Dunkelziffer deutlich höher ist.

\section{Merke}

Korrekte Alarme unterstützen das Personal bei der Versorgung von Patienten, indem sie bei putativ lebensbedrohlichen Situationen informieren. Zahlreiche Fehlalarme sind andererseits eine Belastung von Personal und Arbeitsabläufen. 
- Tab. 1 Alarmquellen auf der Intensivstation [28].

\begin{tabular}{|l|l|}
\hline klinische Alarme & Anzahl \\
\hline Beatmungsgerät & $37,8 \%$ \\
\hline kardiovaskuläres Monitoring & $32,7 \%$ \\
\hline Pulsoxymeter & $14,5 \%$ \\
\hline Kapnometer & $13,5 \%$ \\
\hline
\end{tabular}

\section{Alarmquellen}

In San Francisco wurden auf 5 Intensivstationen mit insgesamt 77 Betten Alarme systematisch analysiert [6]. Es ertönten 187 Alarme pro Bett und Tag. Die häufigsten Alarmquellen waren Beatmungsgeräte und kardiovaskuläres Monitoring, aber zahlreiche weitere Geräte, etwa zur Dialyse-, Kreislauf- oder Vakuumtherapie, sowie Telefone, elektrische Betten und Klimaanlagen können ebenfalls Alarme generieren, die nicht individuell beeinflusst werden können. Schon 1994 gab es über 40 verschiedene Alarme auf einer Intensivstation [3]. 88\% der EKG-Rhythmus-Alarme waren dabei Fehlalarme [18]. Alarmquellen und ihre Bedeutung sind in - Tab. 1 angeführt.

\section{Fehlerquellen}

Die meisten Alarme ertönen, weil vorgegebene Alarmgrenzen über- oder unterschritten werden. Jeder perioperativ tätige Kollege kennt die Folgen von Interferenzen, z.B. durch Elektrokauterisation im EKG, die zu einem Ventrikuläre-Tachykardie-Alarm in der höchsten Kategorie führen können ( $\bullet$ Abb. 1).

Ähnliches kann durch Interferenzen bei der Stimulation zur Generierung motorisch evozierter Potenziale zum Neuromonitoring beobachtet werden ( $\mathbf{A b b} \mathbf{2}$ ). Hierbei entsteht ein Asystoliealarm, ebenfalls ein Alarm in der höchsten Kategorie. Wie auf den Abbildungen beispielhaft zu erkennen, erfolgt hier ein Alarm, obwohl der invasiv gemessene Blutdruck und das periphere Sauerstoffsättigungssignal physiologische Werte anzeigen und damit eine Asystolie sicher ausschließen.

Intensivstationen bieten zudem viele weitere Fehlerquellen. So können Bewegungsartefakte als maligne Herzrhythmusstörungen im EKG fehlgedeutet werden und zum

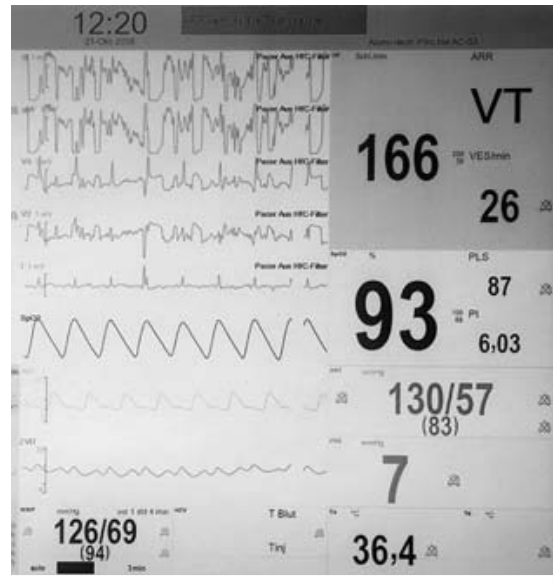

- Abb. 1 Ventrikuläre-TachykardieAlarm durch Interferenzen zwischen Elektrokauterisation und Elektrokardiogramm. Ableitung I zeigt weiterhin $\mathrm{PQRS}$-Komplexe. Der invasiv gemessene Blutdruck sowie die periphere Pulskurve lassen erkennen, dass kein Herzstillstand vorliegen kann.

Alarm führen ( $\triangleright$ Abb. 3 und 4). Außerdem löst Pressen und Husten Druckalarme im Beatmungsgerät und bei extrakorporalen Lungenunterstützungssystemen (ECMO) aus; durch Bewegungen können Sensoren wie der Sauerstoffsättigungsclip verrutschen und falsche Werte anzeigen. Sonnenlicht kann unter bestimmten Bedingungen die Sauerstoffsättigungsmessungen im extrakorporalen Kreislauf einer ECMO stören [19].

\section{Merke}

Bei den meisten Alarmen handelt es sich um Fehlalarme, die eine Desensibilisierung des Personals bewirken. Dies kann die Patientensicherheit gefährden.

\section{Welche Konsequenzen haben Alarme?}

Nicht jeder Alarm bewirkt eine klinische Konsequenz dahingehend, dass ein Mitarbeiter z. B. eine Therapie oder ein medizinisches Gerät anpassen muss. Nach einer älteren Studie ertönten nur 8 von 1455 Alarmen in Folge einer tatsächlich lebensbedrohlichen Situation [20]. Eine neuere Studie aus dem Jahr 2009 zeigt, dass nur $23 \%$ aller Alarme auf einer Intensivstation eine Intervention am Patienten notwendig machen [8]. In einer Untersuchung aus

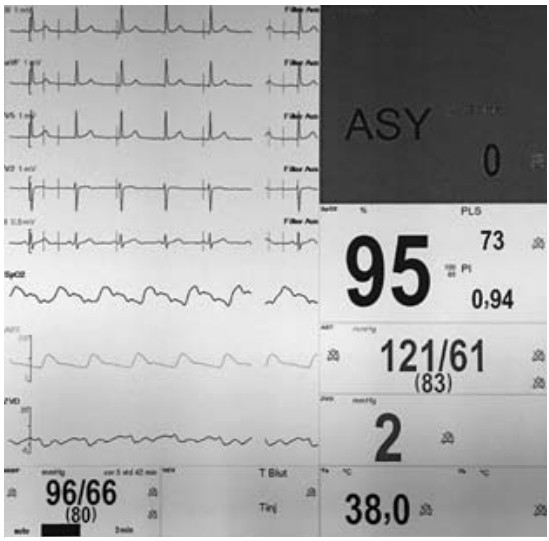

- Abb. 2 Asystoliealarm infolge von Störungen durch motorisch-evozierte Potenziale während einer neurochirurgischen Operation. Trotz vorhandener $\mathrm{PQRS}-$ Komplexe, physiologischem invasiv gemessenem Blutdruck und peripherem Puls meldet das Gerät eine Asystolie. Dies ist Folge eines „unintelligenten“ Alarms.

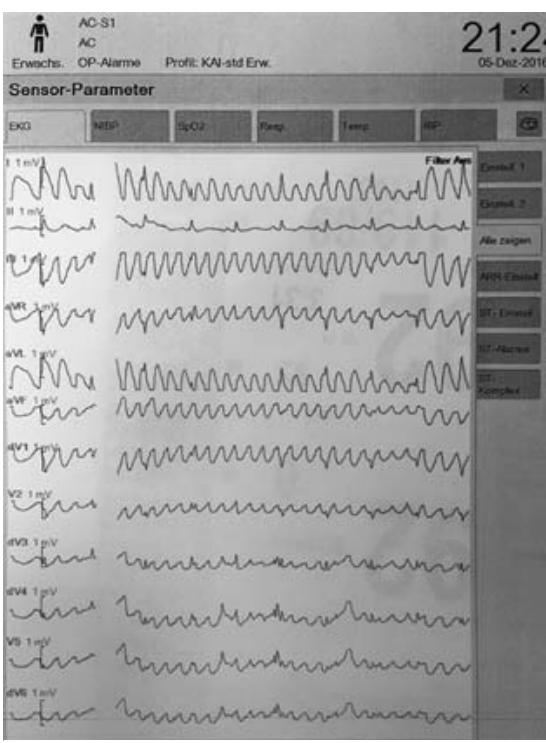

- Abb. 3 Bewegungsartefakte im EKG, die im ersten Augenblick eine maligne Herzrhythmusstörung darstellen können.

einem herzchirurgischen Operationssaal musste bei fast $80 \%$ aller Alarme keine Intervention unternommen werden [21]. Nach einer anderen Untersuchung aus dem Jahr 2010 sind 40\% aller technischen Alarme Fehlalarme, die aber in $98 \%$ mit der höchsten Priorität alarmierten [7], also eine angeblich akut lebensgefährliche Situation suggerieren. 


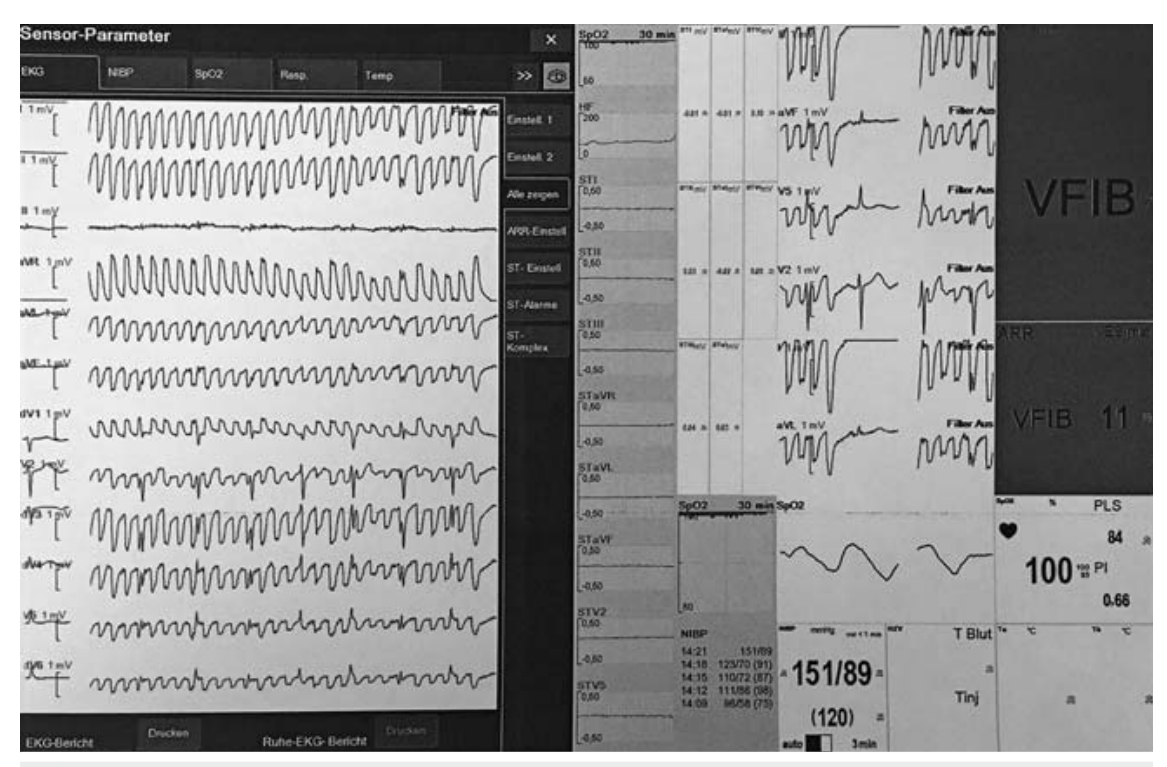

- Abb. 4 Bewegungsartefakte (linke Seite des Bildes) beim wachen Patienten. Nur in Ableitung III ist bei genauer Betrachtung ein PQRS-Komplex zu sehen. Diese Artefakte können etwa dann entstehen, wenn sich ein Rechtshänder die Zähne putzt.

Auch im Operationssaal ist die Situation ähnlich angespannt. Bei 25 herzchirurgischen Operationen ertönten 8975 Alarme, durchschnittlich alle 1,2 Minuten. Allerdings waren nur 70\% dieser Alarme nach videoassistierter Analyse gerechtfertigt, $30 \%$ hingegen Folge von Artefakten. Nur $39 \%$ der wahren, gerechtfertigten Alarme waren indes medizinisch relevant [21]. Auch bei weniger komplexen Operationen ist die Rate an Fehlalarmen vergleichbar hoch [22].

\section{Folgen der Alarmflut}

Eine Pflegekraft auf einer Intensivstation betreut in der Regel zumindest 2 Patienten. Ertönen ein oder mehrere Alarme, muss sie die Arbeit unterbrechen und zunächst prüfen, von welchem Patienten bzw. Zimmer der Alarm stammt und als wie dringlich dieser zu betrachten ist. Unter Umständen muss die Pflegekraft dafür ihre aktuelle Tätigkeit gänzlich unterbrechen und zu dem Patienten oder an einen zentralen Punkt der Intensivstation gehen. Alarme unterbrechen so den Arbeitsablauf und die Patientenversorgung $[2,23]$.

Fehlalarme erhöhen zudem die Arbeitsbelastung unnötig, weil auch für jeden Fehlalarm ein Mitarbeiter seine bisherige Tätigkeit unterbrechen muss, um Ursache und Sicherheitsrelevanz des Alarms zu prüfen $[3,15,24]$. In einer Beobachtungsstudie konnte gezeigt werden, dass das Fehlerrisiko bei der Zubereitung von Medikamenten steigt, je öfter die Mitarbeiter dabei unterbrochen werden [25]. Insbesondere diese Fehlalarme verursachen deshalb „Frustration“ unter den Mitarbeitern $[13,26]$.

Alarme sind zudem einer der Hauptgründe für Lärm auf einer Intensivstation [27, 28]. Lärm ist ein Stressor [14,29,30], der die Kommunikation auf der Station [31-33] und im OP-Raum [29] erschwert. Lärm erhöht den Blutdruck [32] und führte bei Mitarbeitern einer Kinderintensivstation zu Stress, wie durch den Anstieg der Herzfrequenz der Mitarbeiter sowie eine parallel durchgeführte Befragung gezeigt werden konnte [30]. Die Auswertung von Fragebögen ergab ein erhöhtes Risiko bei Mitarbeitern von Intensivstationen für die Entwicklung von Symptomen eines Burnout, wenn diese während der Arbeit durch Lärm gestresst werden [34].

Lärmbelastung führt dazu, dass insbesondere lärmempfindlichen Mitarbeitern häufiger Fehler unterlaufen [35]. Lärmbelastung führt nicht zu einer Gewöhnung, sondern vielmehr auch bei lärmtoleranten Menschen zur Aktivierung sympathoadrenerger Mechanismen und erhöht dadurch das Risiko für Hypertonie und ischämische Herzerkrankungen [36].

\section{Merke}

Alarme unterbrechen die Arbeit der Mitarbeiter und führen zu einem hohen Lärmpegel auf einer Intensivstation.

\section{Die Folgen des Lärms für den Patienten}

Bereits 1910 sagte Robert Koch:

Eines Tages wird der Mensch den Lärm ebenso unerbittlich bekämpfen müssen, wie die Cholera und die Pest [56].

Patienten empfinden ihren Intensivstationsaufenthalt nur sehr selten als erholsam oder angenehm. Ursächlich dafür sind oft Hektik, Lärm und die ständig ertönenden Alarme, außerdem Schmerzen, Schlaflosigkeit, die Unfähigkeit zu kommunizieren und das unangenehme Empfinden aufgrund des Beatmungsschlauchs [37]. Der Aufenthalt auf einer Intensivstation ist eine psychische und körperliche Belastung für die Patienten und ihre Angehörigen [38]. So geben Patienten an, dass ihnen die dortigen Alarme während ihres Intensivaufenthalts Angst machen [39]. Zudem unterbrechen Alarme und hohe Lautstärken den Schlaf der Patienten und erhöhen den Geräuschpegel auf einer Station [37, 40].

\section{INFO}

\section{Lärmobergrenzen}

Die WHO, der International Noise Council und die Environmental Protection Agency fordern für Bereiche des Krankenhauses, in denen Patienten schlafen, untersucht oder behandelt werden, Lärmobergrenzen von $30 \mathrm{~dB}(\mathrm{~A})$ in der Nacht und maximal $45 \mathrm{~dB}(\mathrm{~A})$ am Tag $[30,41]$. Ab $40 \mathrm{~dB}(\mathrm{~A})$ können Arbeiten, die eine hohe Konzentration erfordern, nicht mehr kontinuierlich verlässlich durchgeführt werden [41]. Diese geforderten Lärmpegelobergrenzen werden jedoch auf Intensivstationen regelmäßig deutlich überschritten, mit maximalen Lärmdruckpegeln von $89 \mathrm{~dB}(\mathrm{~A})$ [27]. Die Lärmbelastung auf Intensivstationen ist zudem in den letzten Jahren gestiegen [41]. 
EEG und polysomnografische Messungen während des Schlafes zeigen, dass laute Ereignisse den Schlaf von Intensivpatienten unterbrechen [42]. Schlafunterbrechungen erhöhen die sympathoadrenerge Aktivität, können maligne Herzrhythmusstörungen und Herzstillstände auslösen [43] sowie das Immunsystem durch eine verminderte Anzahl an natürlichen Killerzellen und veränderten Interleukinkonzentrationen hemmen [42].

Lärm erhöht den Stresslevel der Patienten und erschwert die Kommunikation zwischen den Mitarbeitern; die Fehlerrate während der medizinischen Versorgung steigt an [35]. Lärm kann die Heilung verzögern und soll ein Delir auslösen können [30]. Daher hat sich die S3-Leitlinie „Analgesie, Sedierung und Delirmanagement auf der Intensivstation “ indirekt dem Problem angenommen, indem sie eine Reduzierung von Schlafunterbrechungen und Lärmbelästigung zur Delirprophylaxe fordert [44]. Nichtpharmakologische Maßnahmen, wie etwa die Verwendung von Ohrstöpseln zur Reduzierung der Lärmwahrnehmung, werden empfohlen und können die Inzidenz eines Delirs reduzieren [45].

Um die Lärmbelastung und Anzahl der Schlafunterbrechungen zu reduzieren, ist es dringend notwendig, insbesondere die hohe Anzahl an Fehlalarmen zu reduzieren, wobei diese, wie dargestellt, den größten Anteil unter den Alarmen ausmachen.

\section{Merke \\ Der tägliche Lärm auf Intensivstationen stört den Patienten und gefährdet des- sen Gesundheit und Heilung, außerdem belastet er die Mitarbeiter und gefähr- det dadurch die medizinische Versor- gung.}

\section{Lösungsstrategien}

\section{Welche Anforderungen stellen wir an Alarme?}

Alle gefährlichen, insbesondere lebensgefährlichen Situationen müssen erkannt werden, egal ob klinisch oder technisch bedingt [24]. Wir wollen also eine $100 \%$ ige Sensitivität, bei gleichzeitig höchster Spe- zifität. Ein Alarm soll also nur dann ertönen, wenn wirklich eine Gefährdung vorliegt. Im Optimalfall ertönen Alarme vor dem Auftreten einer lebensgefährlichen Situation. Der Alarm muss von den Mitarbeitern gehört und erkannt werden.

Um die Flut an Alarmen, irrelevanten Alarmen und Fehlalarmen zu reduzieren, gibt es verschiedene Ansätze: technische, umweltbedingte und organisatorische.

\section{Technische Ansätze}

\section{Alarmkonfiguration}

20-40\% aller Alarme werden während der Pflege am Patienten verursacht, z. B. durch Bewegungsartefakte, verrutschte Sauerstoffsättigungsclips oder Blutdruckalarme während arterieller Blutabnahmen [7,28, 46]. Ein potenzieller Lösungsansatz für diese Problemkategorie ist die temporäre Stummschaltung der Alarme während der unmittelbaren Pflege am Körper des Patienten. Schon eine 6-sekündige Verzögerung zwischen geräteinterner Problemerkennung und Alarmierung konnte die Hälfte aller Sauerstoffsättigungsalarme verhindern, bei einer Verzögerung von 19 Sekunden waren es sogar 52\% [8]. Auch die Anzahl der Alarme des Beatmungsgeräts konnte man um über ein Drittel reduzieren, wenn der Alarm erst mit einer 19-sekündigen Verzögerung ertönte. Ernste Risiken wurden in dieser Studie hingegen nicht berichtet [3,8]. Diese technischen Veränderungen sind für alle Alarmgrenzen leicht umzusetzen und bereits vorhanden [3]; zudem konnte gezeigt werden, dass individuelle Alarmgrenzen die Häufigkeit der Alarme um bis zu 43\% senken konnten [47].

Eine technische Neuerung ist die persönliche Alarmierung der Bezugspflegekraft des Patienten über ein Smartphone oder ein von Herstellern angebotenes persönliches Alarmierungs-Device. Das Prinzip besteht in der Alarmierung der Bezugspflegekraft mittels persönlicher Nachricht. Auf einer Intensivstation mit 52 Betten konnte in einer Testphase, die auch die Alarmierung der Bezugspflegekraft über ein mobiles Device beinhaltete, die Anzahl der lauten Alarme um 76\% reduziert werden [4]. Ein Push-Nachrichtensystem wurde in einem Krankenhaus-WLAN-System getestet; es alarmierte die Mitarbeiter zu- verlässig und schnell [48]. In einer Studie reagierten auch Anästhesisten auf einen simulierten anästhesiologischen Zwischenfall schneller, wenn sie per Vibrationsalarm statt durch herkömmliches akustisches Monitoring alarmiert wurden [49]. Wichtig zu beachten sind in diesem Zusammenhang die hygienischen Vorschriften und die Zuverlässigkeit der Alarmierung [3] sowie die Einhaltung von Normen wie die europäische Norm EN 60601-1.

\section{Alarmalgorithmen}

Da eine Vielzahl von Alarmen Fehlalarmen zuzuordnen sind, könnte eine Verbesserung geräteinterner Alarmalgorithmen zu einer Verminderung der Alarmhäufigkeit führen [22] - ein Ziel, das sich auch die PhysioNet/Computing in Cardiology Challenge 2015 (Seite 7) setzt. Auf https:// physionet.org werden Open-Source-Software und Datenbanken für an der Lösung technischer Probleme in der Medizin Interessierte angegeben, der Schwerpunkt liegt hier auf der Erfassung physiologischer Messvariablen. Insgesamt erfordert eine Verbesserung der Alarmierung Maßnahmen wie eine Verbesserung der Signalerfassung, neue Wege bei den Alarmalgorithmen und eine exakte Validierung des Alarms [3, 22, 24].

Die meisten Alarme ertönen, weil vorgegebene Alarmgrenzen über- oder unterschritten werden. Die in Abb. 1 und $\mathbf{2}$ dargestellten Fehlalarme kann man durch geräteinterne Hochfrequenzfilter unterdrücken, aber sie zeigen deutlich den Nachteil univariater Alarmierungen. Vorteilhaft wäre eine multivariate Analyse der Vitalparameter, bei der die Überwachungseinheit selbstständig erkennt, dass etwa eine plötzlich auftretende vermeintliche ventrikuläre Tachykardie mit hoher Wahrscheinlichkeit nicht vorliegen kann, während der invasiv gemessene Blutdruck unverändert im normalen Bereich ist. Erkennt der Monitor eine vermeintliche Asystolie bei gleichzeitig noch zu messender peripherer Sättigung und physiologischer Pulskontur, so kann kein Herz-Kreislauf-Stillstand vorliegen, und eine Alarmierung in der höchsten Priorität sollte unterbleiben. Diese intelligente multivariate Analyse würde auch in anderen Bereichen hilfreich sein, um zu verhindern, dass Bewegungsartefakte des wachen Patienten 
EKG-Rhythmus-Alarme im Sinne einer ventrikulären Tachykardie evozieren [3, 50].

Neue Ansätze zur Optimierung der Alarmierungsalgorithmen basieren auf statistischen Methoden und „künstlicher Intelligenz" [3, 22,24]. Blum und Kollegen haben in Salt Lake City eine intelligente netzwerkbasierte Analyse der Vitalparameter integriert. Die Alarmierung erfolgte erst nach länger anhaltender Hypotonie, wenn gleichzeitig der kontinuierlich gemessene Herzindex vermindert war. Die Anzahl der Alarme konnte mithilfe dieses „intelligenten Filters“ im Vergleich zum herkömmlichen, parallel weiter genutzten Standardmonitoring um 99\% gesenkt werden [2].

Ein weiterer Ansatz ist die Vorhersage kritischer Situationen noch vor der Überschreitung von Alarmgrenzen. Realisiert wird dies durch die Auswertung der Trends verschiedener Vitalparameter [3, 24]. Obwohl seit fast 30 Jahren [51] versucht wird, die Alarmflut mithilfe künstlicher Intelligenz und Datenverarbeitungsprogrammen zu reduzieren, sind die Mitarbeiter von Intensivstationen noch immer mit zahlreichen Fehlalarmen konfrontiert [2]. Letztendlich weiß man nicht, ob eine Änderung der Alarmierung, die eine Reduzierung der Fehlalarmrate ermöglicht, die Sensitivität erhöht oder senkt [3].

Selbst wenn Veränderungen der Standardeinstellungen und Schulungen des Pflegepersonals zu einer Reduktion der Alarme um $24 \%$ führte, so konnte die Zufriedenheit der Mitarbeiter trotzdem nicht verbessert werden [23].

\begin{abstract}
Merke
Mithilfe technischer und statistischer Methoden könnte man die Alarmierungsalgorithmen modifizieren, um die Anzahl von Fehlalarmen zu reduzieren.
\end{abstract}

\section{Umwelteinflüsse}

Niedrigere Dauerlärmpegel reduzieren die Belastung des Personals und der Patienten. In der Folge kann auch die Lautstärke der Alarme selbst reduziert werden, weil diese dann nicht mehr die hohen Umgebungslautstärken übertönen müssen. Durch Verwendung moderner Baumaterialien und einer modernen Architektur kann die Absorptionsfähigkeit der Wände erhöht und die akustische Belastung reduziert werden [27]. Bauliche Maßnahmen sollten auch dazu führen, dass Alarme an einem ständig besetzten zentralen Ort der Intensivstation erfasst und beurteilt werden können. Es muss vermieden werden, dass Alarme überhört werden.

\section{Organisatorische Ansätze}

Das auf Stationen mit vielen Überwachungsgeräten eingesetzte Personal muss darin geschult sein, Geräte, Überwachungsumfang und Alarmgrenzen den individuellen Bedürfnissen eines Patienten anzupassen [47]. Die technischen Voreinstellungen der Geräte entsprechen nicht immer den Bedürfnissen aller Patienten.

Personell sollten Intensivstationen möglichst gut ausgestattet sein. Je mehr Patienten eine Pflegekraft zu versorgen hat, insbesondere wenn diese auf verschiedene Zimmer verteilt sind, desto größer die Gefahr, dass auf Alarme nicht zeitnah reagiert werden kann und diese ignoriert werden. Verbesserungen in der personellen Ausstattung von Intensivstationen reduzieren die Arbeitsbelastung der einzelnen Mitarbeiter [52] und ermöglichen so eine Arbeitsteilung [3], sodass beim Ertönen eines Alarms die Tätigkeit der Bezugspflegekraft nicht zwingend unterbrochen, sondern die Überprüfung der Alarmursache an einen freien Mitarbeiter delegiert werden kann. Bliss u. Dunn konnten in einem Experiment zeigen, dass die Reaktion auf Alarme abnimmt, je größer die Arbeitsbelastung ist [53].

Eine lautlose Alarmierung, z. B. per Vibrationsalarm auf ein Alarmierungsgerät der Bezugspflegekraft, würde die Lärmbelastung auf einer Station stark reduzieren [3]. Eine gut ausgebildete Pflegekraft interpretiert zudem bislang die Werte des Monitorings besser als jeder Alarmalgorithmus [3].

Neben dem Monitoring und dem Beatmungsgerät generieren allerdings auch zahlreiche weitere Geräte Alarme, die nicht alle zentral überwacht werden können, aber zur Lärmbelästigung und $\mathrm{Ar}$ beitsbelastung beitragen. Hier sind Lösungsvorschläge der Gerätehersteller einzufordern.
Die Mitarbeiter müssen die Gefahren einer Alarm-Fatigue kennen. Sinnlose Alarme und zu engmaschige Alarmgrenzen müssen hinterfragt und im Team, ggf. gemeinsam mit dem Gerätehersteller, angepasst werden.

Ein multimodaler Ansatz, der den täglichen Wechsel der EKG-Elektroden, tägliches kritisches Hinterfragen der Monitorund Alarmeinstellungen und das zeitweise Unterbrechen des Monitorings von stabiIen Patienten beinhaltet, ermöglicht die Reduktion der Fehlalarmfrequenz anteilig von 95 auf 50\% sowie eine Senkung der durchschnittlichen Alarmfrequenz von 180 auf 40 Alarme proTag [54].

\section{Fazit}

Eine hohe Anzahl und Dichte an Alarmen bewirkt eine Desensibilisierung des Personals, reduziert die Patientensicherheit und führt zu körperlichen sowie psychischen Belastungen der Mitarbeiter, Patienten und deren Angehöriger während des Besuchs. Durch statistische und technische Methoden könnten die geräteinternen Algorithmen der Alarmierung modifiziert werden. Außerdem vermag ein modernes Alarmmanagement das Personal individuell zu warnen. Durch Optimierung der baulichen und technischen Ausstattung und durch Schulung der Mitarbeiter können Fehlalarme und schließlich AlarmFatigue reduziert werden. Dies sind allerdings zum Teil kostenintensive Investitionen, die multimodale Lösungsansätze erfordern sowie eine enge Zusammenarbeit mit Geräteherstellern, Krankenhausleitung und den Mitarbeitern.

Tipps zur Reduktion von Alarm-Fatigue, Fehlalarmen und für ein modernes Alarmmanagement umfassen:

- tägliches Wechseln der EKG-Elektroden zur Verbesserung der Signalqualität, reduziert die Fehlalarmrate um 46\% [55].

- Schulung des Personals und individuelle, patientenbezogene Einstellung der Alarmgrenzen reduzieren die Monitoralarme um $43 \%$ [47].

- Optimierung der personellen Besetzung von Stationen mit intensivem Geräteeinsatz 
- Tab. 2 Faktoren zur Minimierung von Alarm-Fatigue [32].

\begin{tabular}{|c|c|c|}
\hline \multicolumn{2}{|l|}{ Human Factors } & technische Aspekte \\
\hline $\begin{array}{l}\text { Training des } \\
\text { Personals }\end{array}$ & $\begin{array}{l}\text { - Einstellung der Alarmgrenzen } \\
\text { - tägliches Wechseln der EKG-Elektroden } \\
\text { - individuelle Alarmgrenzen }\end{array}$ & \multirow{3}{*}{$\begin{array}{l}\text { - Signalqualität } \\
\text { - Artefakte } \\
\text { - Alarmalgorithmen } \\
\text { - Fehlalarmrate } \\
\text { - moderne Alarmie- } \\
\text { rungsmethoden }\end{array}$} \\
\hline Umweltfaktoren & - Reduzierung der Lärmbelastung & \\
\hline $\begin{array}{l}\text { Arbeitsbelastung } \\
\text { des Personals }\end{array}$ & $\begin{array}{l}\text { - zahlenmäßiges Verhältnis von Patienten } \\
\text { zu Pflegepersonal }\end{array}$ & \\
\hline
\end{tabular}

- Reduzierung der allgemeinen Lärmbelastung

- Einsatz von Ohrstöpseln für die Patienten während deren Ruhezeiten

- Informationen für Patienten und deren Angehörige

- individuelle Alarmierung des Bezugspflegepersonals

- Sensibilisierung des Personals für die Gefahren von „Alarm-Fatigue“

- Sicherstellung, dass Alarme nicht aus räumlichen Gründen überhört werden

Maßnahmen, mit denen das Risiko einer Alarm-Fatigue reduziert werden kann, sind in $>$ Tab. 2 zusammengefasst.

\section{KERNAUSSAGEN}

- Zunehmende Technisierung erhöht die Zahl an Alarmen.

- Die meisten Alarme sind Fehlalarme.

- Fehlalarme bewirken eine Desensibilisierung der Mitarbeiter.

- „Wer einmal lügt, dem glaubt man nicht“ - dies gilt auch für Alarme!

- Laute Alarme schädigen den Patienten.

- Fehlalarme unterbrechen die Arbeit und führen zu Frustration.

- Fehlalarme müssen reduziert werden.

\section{Interessenkonflikt}

Die Autoren erklären, dass kein Interessenkonflikt besteht.

\section{Über die Autoren}

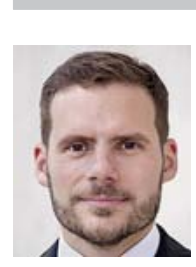

\section{Benedikt Lorenz} an der Klinik für Anästhesiologie und Intensivmedizin, Universitätsklinikum Essen. 2008-2013 Studium der Medizin an der Medizinischen Universität Graz. Seit 2014 Assistenzarzt an der Universitätsklinik für Anästhesiologie und Intensivmedizin in Essen. Weiterbildungen/Fortbildungen in: PALS, ATLS, Fachkunde Rettungsdienst, Echokardiografie und UItraschall

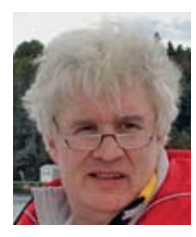

\section{Jürgen Peters}

Prof. Dr. med., Direktor der Klinik für Anästhesiologie und Intensivmedizin, Universitätsklinikum Essen. 1984 Facharztanerkennung für Anästhesiologie, Ausbildung in Duisburg, Düsseldorf und The John Hopkins University Medical School Baltimore, $1990 \mathrm{Ha}$ bilitation und Erteilung der Venia Legendi, 1992 Leitender Oberarzt am Institut für Klinische Anästhesiologie, Universität Düsseldorf. Seit 1996 Direktor der Klinik für Anästhesiologie und Intensivmedizin des Universitätsklinikums Essen.

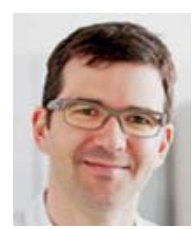

\section{Ulrich Frey} Erlangung des Doktorgrades der Medizin, seit 2010 Oberarzt, 2011 Habilitation und Venia Legendi im Fach Anästhesiologie und Intensivmedizin. Seit 2013 Geschäftsführender Oberarzt an der Klinik für Anästhesiologie und Intensivmedizin, Universitätsklinikum Essen, seit 2014 Leitender Oberarzt Anästhesiologie, Bereich Herz-Thorax und kardiovaskuläre Chirurgie. Zusatzqualifikation/-bezeichnung u. a. in Intensivmedizin und Notfallmedizin.
Dr. med. univ., Assistenzarzt

Prof. Dr. med., MHBA. 2001
Dr. med. univ. Benedikt Lorenz

Klinik für Anästhesiologie und Intensivmedizin

Universität Duisburg-Essen und

Universitätsklinikum Essen (AöR)

Hufelandstraße 55

45147 Essen

Benedikt.Lorenz@uk-essen.de

\section{Literatur}

[1] Westenskow DR, Orr JA, Simon FH et al. Intelligent alarms reduce anesthesiologist's response time to critical faults. Anesthesiology 1992; 77: 1074-1079

[2] Blum JM, Kruger GH, Sanders KL et al. Specificity improvement for network distributed physiologic alarms based on a simple deterministic reactive intelligent agent in the critical care environment. J Clin Monit Comput 2009; 23: 21-30

[3] Borowski M, Görges M, Fried R et al. Medical device alarms. Biomed Tech (Berl) 2011; 56: 73-83

[4] Jahrsdoerfer M. Case study: reducing interruption fatigue through improved alarm support. Biomed Instrum Technol 2016; 50: 109-113

[5] Chambrin MC. Alarms in the intensive care unit: how can the number of false alarms be reduced? Crit Care 2001; 5: 184-188

[6] Drew BJ, Harris P, Zègre-Hemsey JK et al. Insights into the problem of alarm fatigue with physiologic monitor devices: a comprehensive observational study of consecutive in tensive care unit patients. PLoS One 2014; 9: e110274

[7] Siebig S, Kuhls S, Imhoff $M$ et al. Intensive care unit alarms-how many do we need? Crit Care Med 2010; 38: 451-456

[8] Görges M, Markewitz BA, Westenskow DR. Improving alarm performance in the medical intensive care unit using delays and clinical context. Anesth Analg 2009; 108: 1546-1552

[9] Joint Commission. Medical device alarm safety in hospitals. Sentinel Event Alert 2013; 50: 1-3

[10] Rayo MF, Moffatt-Bruce SD. Alarm system management: evidence-based guidance encouraging direct measurement of informativeness to improve alarm response. BM] Qual Saf 2015; 24: 282-286

[11] Meredith C, Edworthy ]. Are there too many alarms in the intensive care unit? An overview of the problems. I Adv Nurs 1995; 21: 15-20

[12] Chambrin MC, Ravaux P, Calvelo-Aros D et al. Multicentric study of monitoring alarms in the adult intensive care unit (ICU): a descriptive analysis. Intensive Care Med 1999; 25: 1360-1366 
[13] Christensen M, Dodds A, Sauer ] et al. Alarm setting for the critically ill patient: a descriptive pilot survey of nurses' perceptions of current practice in an Australian Regional Critical Care Unit. Intensive Crit Care Nurs 2014; 30: 204-210

[14] Clifford GD, Silva I, Moody B et al. False alarm reduction in critical care. Physiol Meas 2016; 37: E5-E23

[15] Sowan AK, Tarriela AF, Gomez TM et al. Nurses' perceptions and practices toward clinical alarms in a transplant cardiac intensive care unit: exploring key issues leading to alarm fatigue. JMIR Human Factors 2015; 2: e3

[16] Mitka M. Joint commission warns of alarm fatigue: multitude of alarms from monitoring devices problematic. JAMA 2013; 309: 2315-2316

[17] ECRI Institute. Top 10 Health Technology Hazards for 2015. Health Devices 2014; 43: $1-33$

[18] Drew B], Harris P, Zègre-Hemsey JK et al. Insights into the problem of alarm fatigue with physiologic monitor devices: a comprehensive observational study of consecutive intensive care unit patients. PLoS One 2014; 9: e110274

[19] Dirkmann D, Kehren C], Adamzik M. Recurrent false alarm of extracorporeal membrane oxygenation at high noon. Eur ] Anaesthesiol 2012; 29: 602-604

[20] O'Carroll TM. Survey of alarms in an intensive therapy unit. Anaesthesia 1986; 41: 742-744

[21] Schmid F, Goepfert MS, Kuhnt D et al. The wolf is crying in the operating room. Anesth Analg 2011; 112: 78-83

[22] Schmid F, Goepfert MS, Reuter DA. Patient monitoring alarms in the ICU and in the operating room. Crit Care 2013; 17: 216

[23] Sowan AK, Gomez TM, Tarriela AF et al. Changes in default alarm settings and standard in-service are insufficient to improve alarm fatigue in an intensive care unit: a pilot project. JMIR Human Factors 2016; 3: e1

[24] Imhoff M, Kuhls S. Alarm algorithms in critical care monitoring. Anesth Analg 2006; 102: $1525-1537$

[25] Westbrook JI, Woods A, Rob Ml et al. Association of interruptions with an increased risk and severity of medication administration errors. Arch Intern Med 2010; 170: 683-690

[26] Korniewicz DM, Clark T, David Y. A national online survey on the effectiveness of clinical alarms. Am J Crit Care 2008; 17: 36-41

[27] MacKenzie DJ, Galbrun L. Noise levels and noise sources in acute care hospital wards. Build Serv Eng Res Technol 2007; 28: 117131

[28] Chambrin MC, Ravaux P, Calvelo-Aros D et al. Multicentric study of monitoring alarms in the adult intensive care unit (ICU): a descriptive analysis. Intensive Care Med 1999; 25: 1360-1366
[29] Hasfeldt D, Laerkner E, Birkelund R. Noise in the operating room - what do we know? A review of the literature. J Perianesth Nurs 2010; 25: 380-386

[30] Morrison WE, Haas EC, Shaffner DH et al. Noise, stress, and annoyance in a pediatric intensive care unit. Crit Care Med 2003; 31: 113-119

[31] Weinger MB, Smith NT. Vigilance, alarms, and integrated monitoring systems. In: Ehrenwerth J, Eisenkraft JB, editors. Anesthesia equipment: principles and applications. Malvern, PA: Mosby Year Book; 1993. 350-384

[32] Solet JM, Barach PR. Progress in pediatric cardiology. Progr Pediatr Cardiol 2012; 33: 8590

[33] Edworthy ]. Medical audible alarms: a review. J Am Med Inform Assoc 2013; 20: 584-589

[34] Topf M, Dillon E. Noise-induced stress as a predictor of burnout in critical care nurses. Heart Lung 1988; 17: 567-574

[35] Belojević G, Ohrström E, Rylander R. Effects of noise on mental performance with regard to subjective noise sensitivity. Int Arch Occup Environ Health 1992; 64: 293-301

[36] Ising H, Kruppa B. Zum gegenwärtigen Erkenntnisstand der Lärmwirkungsforschung: Notwendigkeit eines Paradigmenwechsels. Umweltmed Forsch Prax 2001; 6: 181

[37] Cornock MA. Stress and the intensive care patient: perceptions of patients and nurses. J Adv Nurs 1998; 27: 518-527

[38] Jones C, Skirrow P, Griffiths RD et al. Posttraumatic stress disorder-related symptoms in relatives of patients following intensive care. Intensive Care Med 2004; 30: 456-460

[39] Yava A, Tosun N, Ünver V et al. Patient and nurse perceptions of stressors in the intensive care unit. Stress Health 2011; 27: e36e47

[40] Cochran J, Ganong LH. A comparison of nurses' and patients' perceptions of intensive care unit stressors. J Adv Nurs 1989; 14 : 1038-1043

[41] Konkani A, Oakley B. Noise in hospital intensive care units. J Crit Care 2012; 27: 522.e1522.e9

[42] Xie H, Kang J, Mills GH. Clinical review: The impact of noise on patients' sleep and the effectiveness of noise reduction strategies in intensive care units. Crit Care 2009; 13: 208

[43] Miner SE, Pahal D, Nichols L et al. Sleep disruption is associated with increased ventricular ectopy and cardiac arrest in hospitalized adults. Sleep 2016; 39: 927-935

[44] Deutsche Gesellschaft für Anästhesiologie und Intensivmedizin e.V. (DGAI), Deutsche Interdisziplinäre Vereinigung für Intensivund Notfallmedizin (DIVI). S3-Leitlinie „Analgesie, Sedierung und Delirmanagement in der Intensivmedizin“, AWMF-Register Nr. 001/012, Stand: 31.08.2015, gültig bis 30. 08. 2020. Im Internet: http://www.awmf. org/uploads/tx_szleitlinien/001-012I_S3_
Analgesie_Sedierung_Delirmanagement_ Intensivmedizin_2015-08_01.pdf; Stand: 23.06.2016

[45] Van Rompaey B, Elseviers MM, Van Drom W et al. The effect of earplugs during the night on the onset of delirium and sleep perception: a randomized controlled trial in intensive care patients. Crit Care 2012; 16: R73

[46] Ruskin KJ, Hueske-Kraus D. Alarm fatigue: impacts on patient safety. Curr Opin Anaesthesiol 2015; 28: 685-690

[47] Graham KC, Cvach M. Monitor alarm fatigue: standardizing use of physiological monitors and decreasing nuisance alarms. Am J Crit Care 2010; 19: 28-34

[48] Rothman BS, Dexter F, Epstein RH. Communication Latencies of Apple Push Notification Messages Relevant for Delivery of Time-Critical Information to Anesthesia Providers. Anesth Analg 2013; 117: 398-404

[49] Ford S, Daniels J, Lim J et al. A novel vibrotactile display to improve the performance of anesthesiologists in a simulated critical incident. Anesth Analg 2008; 106: 1182-1188

[50] Feldman JM, Ebrahim MH, Bar-Kana I. Robust sensor fusion improves heart rate estimation: clinical evaluation. J Clin Monit 1997; 13: $379-384$

[51] Carson ER. Artificial intelligence in critical care medicine: EC COST 13 project. J Clin Monit Comput 1988; 5: 251

[52] Aiken LH, Sloane DM, Cimiotti JP et al. Implications of the California Nurse Staffing Mandate for other states. Health Services Res 2010; 45: 904-921

[53] Bliss JP, Dunn MC. Behavioural implications of alarm mistrust as a function of task workload. Ergonomics 2000; 43: 1283-1300

[54] Dandoy CE, Davies SM, Flesch L et al. A teambased approach to reducing cardiac monitor alarms. Pediatrics 2014; 134: e1686-e1689

[55] Cvach MM, Biggs M, Rothwell KJ et al. Daily electrode change and effect on cardiac monitor alarms. J Nursing Care Quality 2013; 28: 265-271

[56] Schmidt L. Im Internet: http://www.faz.net/ aktuell/feuilleton/auswirkungen-von-laermwarum-tun-wir-uns-das-an-11865692.html, Frankfurter Allgemeine Zeitung vom 24.08.2014; Stand: 11.10.2016

Bibliografie

DOI https://doi.org/10.1055/s-0042-118618 Anästhesiol Intensivmed Notfallmed Schmerzther 2017; 52: 564-570 @ Georg Thieme Verlag KG Stuttgart · New York | ISSN 0939-2661 